\title{
Limits to dark matter properties from a combined analysis of MAGIC and Fermi-LAT observations of dwarf satellite galaxies
}

\author{
Javier Rico \\ Institut de Física d'Altes Energies (IFAE), E-08193 Bellaterra, Spain \\ E-mail: jrico@ifae.es

\section{Matthew Wood*} \\ Kavli Institute for Particle Astrophysics and Cosmology, \\ Department of Physics and SLAC National Accelerator Laboratory \\ E-mail: mdwoodeslac.stanford.edu
}

\section{Alex Drlica-Wagner}

Center for Particle Astrophysics, Fermi National Accelerator Laboratory

E-mail: kadrlicalfnal.gov

\section{Jelena Aleksić}

Institut de Física d'Altes Energies (IFAE), E-08193 Bellaterra, Spain

E-mail: jelena@ifae.es

\section{for the MAGIC ${ }^{\dagger}$ and the Fermi-LAT ${ }^{\ddagger}$ Collaborations}

\begin{abstract}
We present the first MAGIC/Fermi-LAT joint search for dark matter annihilation gamma-ray signals from dwarf satellite galaxies. We combine 158 hours of observations of Segue 1 by MAGIC with 6-years observations of 15 dwarf satellite galaxies by the Fermi-LAT. We obtain limits on the annihilation cross-section for dark matter particle masses between $10 \mathrm{GeV}$ and $100 \mathrm{TeV}-$ the widest mass range ever explored by a coherent and comprehensive analysis. Our new inclusive analysis approach is completely generic, and we propose to use it to perform a global, sensitivityoptimized dark matter search by combining data from present and future gamma-ray and neutrino detectors.
\end{abstract}

The 34th International Cosmic Ray Conference,

30 July- 6 August, 2015

The Hague, The Netherlands

\footnotetext{
*Speaker.

${ }^{\dagger}$ http://magic.mpp.mpg.de

${ }^{\ddagger} \mathrm{http} / / /$ www-glast.stanford.edu
} 


\section{Introduction}

Dark matter (DM) distributes in the Universe in halos that host galaxy clusters, galaxies and galactic DM "clumps". A promising way to identify the nature of DM and measure its properties is to search for the Standard Model (SM) particles produced in its annihilation or decay at these sites. Gamma rays and neutrinos are ideal messengers for directional DM searches, since they are the only stable neutral SM particles, and can thus travel from their production sites to Earth unaffected by magnetic deflection.

Current gamma-ray instruments like the Fermi-LAT in space, the ground-based Cherenkov telescopes MAGIC, VERITAS and H.E.S.S., and the new-generation water Cherenkov detector HAWC, as well as neutrino telescopes like IceCube and Antares, are sensitive to overlapping and complementary DM particle mass ranges (from $\sim 1 \mathrm{GeV}$ to $\sim 100 \mathrm{TeV}$ ). All of these instruments have dedicated programs to look for DM signals coming from, e.g., the Galactic center and halo $[1,2,3,4,5,6,7]$, galaxy clusters $[8,9,10,11,12]$, or dwarf spheroidal satellite galaxies (dSphs) of the Milky Way (MW) $[13,14,15,16]$.

The universality of DM properties allows the combination of data from different experiments and/or observational targets into a global, sensitivity-optimized search [17]. For a given DM particle model, a joint likelihood function can be written as the product of the partial contributions from each of the measurements/instruments. The advantage of such an approach is that the details of each experiment do not need to be combined or averaged. We have implemented this analysis framework, applicable to observations from gamma-ray and neutrino instruments, and applied it to the MAGIC and Fermi-LAT observations of dSphs.

The MW dSphs are associated to the Galactic DM sub-haloes, predicted by N-body cosmological simulations, that have attracted enough baryonic mass to start stellar activity (other sub-halos may remain completely dark). MW dSphs have very high mass-to-light ratios, being the most DM-dominated systems known so far [18]. MW dSphs also have the advantage of being free of astrophysical gamma-ray sources and, while they are relatively close, they still appear as quasipoint-like sources for gamma-ray and neutrino telescopes, with relatively high expected fluxes. In addition, the possibility of determining the DM distribution and its uncertainty using a common methodology [19] allows a straightforward combination of their observations into a sensitivityoptimized global analysis.

In this paper, we present our new global analysis framework and the results of applying it to MAGIC and Fermi-LAT observations of dSphs.

\section{MAGIC and Fermi-LAT data samples}

The Florian Goebel MAGIC telescopes are located at the Roque de los Muchachos Observatory $\left(28.8^{\circ} \mathrm{N}, 17.9^{\circ} \mathrm{W} ; 2200 \mathrm{~m}\right.$ above sea level), at the Canary Island of La Palma (Spain). MAGIC is a system of two telescopes that detect Cherenkov light produced by the atmospheric showers initiated by cosmic particles entering the Earth atmosphere. Cherenkov images of the showers are projected by MAGIC reflectors onto the photo-multiplier tube (PMT) cameras, and are used to reconstruct the calorimetric and spatial properties of the primary particle, as well as for its identification. Thanks to its large reflectors (17 meter diameter), plus its high-quantum-efficiency and 
low-noise PMTs, MAGIC achieves high sensitivity to Cherenkov light, hence low energy threshold. The MAGIC telescopes are able to detect cosmic gamma rays in the very-high-energy domain, i.e. in the range between $\sim 50 \mathrm{GeV}$ and $\sim 50 \mathrm{TeV}$.

For our study, we use MAGIC data corresponding to 158 hours of observations of Segue 1 [13], the deepest observations of any dSph by any Cherenkov telescope. The data were taken between 2011 and 2013 [20].

The Fermi-LAT is a pair-conversion telescope sensitive to gamma rays in the range from $20 \mathrm{MeV}$ to more than $300 \mathrm{GeV}$ [21]. With its large field of view (2.4 sr), the Fermi-LAT is able to efficiently survey the entire sky. Indeed, since its launch in August 2008, the Fermi-LAT has primarily operated in a survey mode that scans the entire sky every 3 hours. The survey-mode exposure coverage is fairly uniform over the sky with variations of at most $30 \%$ with respect to the average exposure. The Fermi-LAT source sensitivity, which is limited by the intensity of diffuse backgrounds, shows larger variations but is relatively constant at high galactic latitudes $\left(b>10^{\circ}\right)$.

In this work, we use a Fermi-LAT data sample corresponding to 6 years of observations of $15 \mathrm{dSphs}$, processed with the latest (Pass 8) data analysis [16]. Events are selected with energies between $500 \mathrm{MeV}$ and $500 \mathrm{GeV}$ in a $10^{\circ} \times 10^{\circ}$ region of interest (ROI) centered on each $\mathrm{dSph}$. LAT likelihoods for a given DM model are constructed from the bin-by-bin likelihoods of [16] and do not involve any reanalysis of the LAT photon data.

\section{Analysis}

\subsection{Dark Matter annihilation flux}

The gamma-ray (or neutrino) flux produced by DM annihilation in a given target and detectable at Earth by an instrument observing a field of view $\Delta \Omega$ is given by:

$$
\frac{d \Phi}{d E}(\Delta \Omega)=\frac{1}{4 \pi} \frac{\langle\sigma v\rangle J(\Delta \Omega)}{2 m_{\mathrm{DM}}^{2}} \frac{d N}{d E}
$$

where $m_{\mathrm{DM}}$ is the mass of the DM particle, $\langle\sigma v\rangle$ the thermally-averaged annihilation cross section, $d N / d E$ the average gamma-ray spectrum per annihilation reaction (for neutrino this term includes the oscillation probability between target and Earth), and

$$
J(\Delta \Omega)=\int_{\Delta \Omega} d \Omega \int_{\text {1.o.s. }} d l \rho^{2}(l, \Omega)
$$

is the so-called astrophysical factor (or simply J-factor), with $\rho$ being the DM density, and the integrals running over $\Delta \Omega$ and the line of sight (l.o.s.), respectively.

Using PYTHIA simulation package version 8.205 [22], we have computed the average gammaray spectra per annihilation process $(d N / d E)$ for a set of DM particles of masses between $10 \mathrm{GeV}$ and $100 \mathrm{TeV}$, annihilating into SM pairs $b \bar{b}$ and $\tau^{+} \tau^{-}$. For each channel and mass, we average the gamma-ray spectrum resulting from $10^{7}$ decay events of a generic resonance with mass $2 \times m_{\mathrm{DM}}$ into the specified pairs. For each simulated event, we trace all the decay chains, including the muon radiative decay ( $\mu^{-} \rightarrow e^{-} \bar{v}_{e} v_{\mu} \gamma$, not active in PYTHIA by default), down to stable particles.

For the computation of the J-factors we follow the approach by Martinez [19]. The distribution of DM in the halos of the different dSphs are parameterized following a Navarro-Frenk-White 
profile (NFW) [23]:

$$
\rho(r)=\frac{\rho_{0} r_{s}^{3}}{r\left(r_{s}+r\right)^{2}}
$$

where $r_{s}$ and $\rho_{0}$ are the NFW scale radius and characteristic density, respectively, and are determined from a fit to the dSph stellar density and velocity dispersion profiles. The properties of the dSphs used in our analysis, including the J-factors and their uncertainties, can be found in [16].

We use templates for the DM emission in each dSph normalized to its J-factor integrated to a radius of $0.5^{\circ}$ from the halo center $\left(J_{\mathrm{obs}, \mathrm{i}}\right)$. The $0.5^{\circ}$ integration region encompasses more than $90 \%$ of the annihilation flux for our dSph halo models which have halo scale radii between $0.1^{\circ}$ and $0.4^{\circ}$. In the LAT analysis the intensity templates are used to construct a three-dimensional model for the expected DM signal as a function of space and energy within the $10^{\circ} \times 10^{\circ}$ ROI centered on each $\mathrm{dSph}$. The MAGIC analysis uses a one-dimesional likelihood for the photon energy distribution within a signal aperture of radius $0.122^{\circ}$. The observable flux in the MAGIC analysis of Segue 1 is therefore reduced by a factor of $\sim 1.6$ with respect to the LAT analysis of the same target. However we note that because the signal aperture is matched to the angular size of the MAGIC PSF, this truncation has a negligible impact on the sensitivity of the analysis.

\subsection{Likelihood analysis}

For each considered annihilation channel and DM particle mass, we compute the profile likelihood ratio as a function of $\langle\sigma v\rangle$ :

$$
\lambda_{P}(\langle\sigma v\rangle \mid \mathscr{D})=\frac{\mathscr{L}(\langle\sigma v\rangle ; \hat{\hat{v}} \mid \mathscr{D})}{\mathscr{L}(\langle\widehat{\sigma v}\rangle ; \hat{\boldsymbol{v}} \mid \mathscr{D})},
$$

with $\mathscr{D}$ representing the data samples and $\boldsymbol{v}$ the nuisance parameters. $\langle\widehat{\sigma v}\rangle$ and $\hat{\boldsymbol{v}}$ are the values maximizing the joint likelihood function $(\mathscr{L})$, and $\hat{\hat{\boldsymbol{v}}}$ the value that maximizes $\mathscr{L}$ for a given value of $\langle\sigma v\rangle$. The likelihood function can be written as:

$$
\mathscr{L}(\langle\sigma v\rangle ; \boldsymbol{v} \mid \mathscr{D})=\prod_{i=1}^{N_{\text {target }}} \mathscr{L}_{i}\left(\langle\sigma v\rangle ; J_{i}, \boldsymbol{\mu}_{i} \mid \mathscr{D}_{i}\right) \cdot \mathscr{J}\left(J_{i} \mid J_{\mathrm{obs}, i}, \sigma_{i}\right)
$$

with the index $i$ running over the different targets (dSphs in our case); $J_{i}$ is the J-factor for the corresponding target (see Equation 3.2); $\mu_{i}$ denotes any additional nuisance parameters; and $\mathscr{D}_{i}$ the target-related input data. $\mathscr{J}$ is the likelihood for $J_{i}$, given measured $\log _{10}\left(J_{\mathrm{obs}, i}\right)$ and its uncertainty $\sigma_{i}[16]$, i.e.:

$$
\mathscr{J}\left(J_{i} \mid J_{\mathrm{obs}, \mathrm{i}}, \sigma_{i}\right)=\frac{1}{\ln (10) J_{\mathrm{obs}, \mathrm{i}} \sqrt{2 \pi} \sigma_{i}} \times e^{-\left(\log _{10}\left(J_{i}\right)-\log _{10}\left(J_{\mathrm{obs}, \mathrm{i}}\right)\right)^{2} / 2 \sigma_{i}^{2}}
$$

The likelihood function for a particular target $\left(\mathscr{L}_{i}\right)$ can in turn be written as the product of the likelihoods for different instruments (represented by the index $j$ ), i.e.:

$$
\mathscr{L}_{i}\left(\langle\sigma v\rangle ; J_{i}, \boldsymbol{\mu}_{i} \mid \mathscr{D}_{i}\right)=\prod_{j=1}^{N_{\text {instrument }}} \mathscr{L}_{i j}\left(\langle\sigma v\rangle ; J_{i}, \boldsymbol{\mu}_{i j} \mid \mathscr{D}_{i j}\right)
$$

where $\boldsymbol{\mu}_{i j}$ and $\mathscr{D}_{i j}$ represent the nuisance parameters and input data sample for the given target $i$ and instrument $j$. 
Equations 3.4, 3.5 and 3.7 are generic, i.e. they are valid for any set of instruments and observed targets. In addition, they allow merging of the results from different instruments and targets, starting from tabulated values of $\mathscr{L}_{i j}$ vs. $\langle\sigma v\rangle$ for a fixed value of $J_{i}$ and profiled with respect to $\boldsymbol{\mu}_{i j}$. These values can be produced and shared by the different experiments without the need of releasing or sharing any of the internal information used to produce them.

For this work, we use the Fermi-LAT likelihood values vs. energy flux, tabulated for different targets and in energy bins, and released by the Fermi-LAT Collaboration [16]. In the case of MAGIC, the likelihood is obtained following the method described in Refs. [17] and [13].

\section{Results}

We compute one-sided, 95\% confidence level upper limits to $\langle\sigma v\rangle$ by numerically solving the equation $-2 \ln \lambda_{P}\left(\langle\sigma v\rangle_{2.71} \mid \mathscr{D}\right)=2.71$, for $\langle\sigma v\rangle_{2.71}$. We consider $\lambda_{P}$ restricted to non-negative $\langle\sigma v\rangle$ values only.

Figure 1 shows the $95 \%$ confidence level limits to $\langle\sigma v\rangle$ for DM particles with masses between $10 \mathrm{GeV}$ to $100 \mathrm{TeV}$ annihilating into SM pairs $\left(b \bar{b}\right.$ and $\left.\tau^{+} \tau^{-}\right)$obtained from the combination of Fermi-LAT (15 dSphs and Segue 1 alone) and MAGIC Segue 1 observations. The $68 \%$ and 95\% containment bands are computed from the distribution of upper limits obtained from 300 analysis realizations consisting of Fermi-LAT observations of empty fields of view combined with MAGIC fast simulations (assuming in both cases equal exposures as for the real data), and J-factors randomly selected according to the probability density function (PDF) in Equation 3.6. The blank fields constituting the LAT realizations were selected by choosing randomy sky positions with $|b|>30^{\circ}$ centered at least $0.5^{\circ}$ from a source in the 3 FGL catalog. MAGIC fast simulations consist of a set of event energies randomly generated from the background PDF (see Ref. [13] for details) for both signal and background regions.

We find no positive signal of DM in our data sample. As expected, limits in the low and high ends of the considered mass range are dominated by Fermi-LAT and MAGIC observations, respectively, where the combined limits coincide with the individual ones. The combination provides a significant improvement in the range between $\sim 1$ and $\sim 30 \mathrm{TeV}$ (for $b \bar{b}$ ) or $\sim 0.2$ and $\sim 2 \mathrm{TeV}$ (for $\tau^{+} \tau^{-}$), with a maximum improvement of the combined limits with respect to the individual ones by a factor $\sim 2$ at a mass of $500 \mathrm{GeV}$ (for $b \bar{b}$ ) and $3 \mathrm{TeV}$ (for $\tau^{+} \tau^{-}$).

MAGIC individual results shown here may differ from those presented in Ref. [13] by up to a factor $\sim 4$, which needs a dedicated explanation. First, we note that the data, instrument response functions, and likelihood functions are identical in both works. Aside from enlarging the explored DM mass range, and in order to homogenize MAGIC and Fermi-LAT analyses, we have introduced the following differences between the two works: $i$ ) the J-factor for MAGIC cut of $J\left(0.122^{\circ}\right)=2.2 \times 10^{19} \mathrm{GeV}^{2} \mathrm{~cm}^{-5}$ (following Ref. [19] and assuming an NFW DM density profile); ii) include the statistical uncertainties in the determination of the J-factor; iii) use the FermiLAT prescription for limits close to bounds of the physical region $(\langle\sigma v\rangle\rangle=0$ ) (here we restrict the function $-2 \ln \lambda_{P}(\langle\sigma v\rangle \mid \mathscr{D})$ to $\langle\sigma v\rangle \geq 0$, whereas in previous MAGIC results $\lambda_{P}$ was also computed for negative $\langle\sigma v\rangle$ values, and the quoted limit was $\langle\sigma v\rangle_{\text {svt }}=\langle\sigma v\rangle_{2.71}-\langle\widehat{\sigma v}\rangle$, whenever $\langle\widehat{\sigma v}\rangle\langle 0$, which is a more conservative choice). 

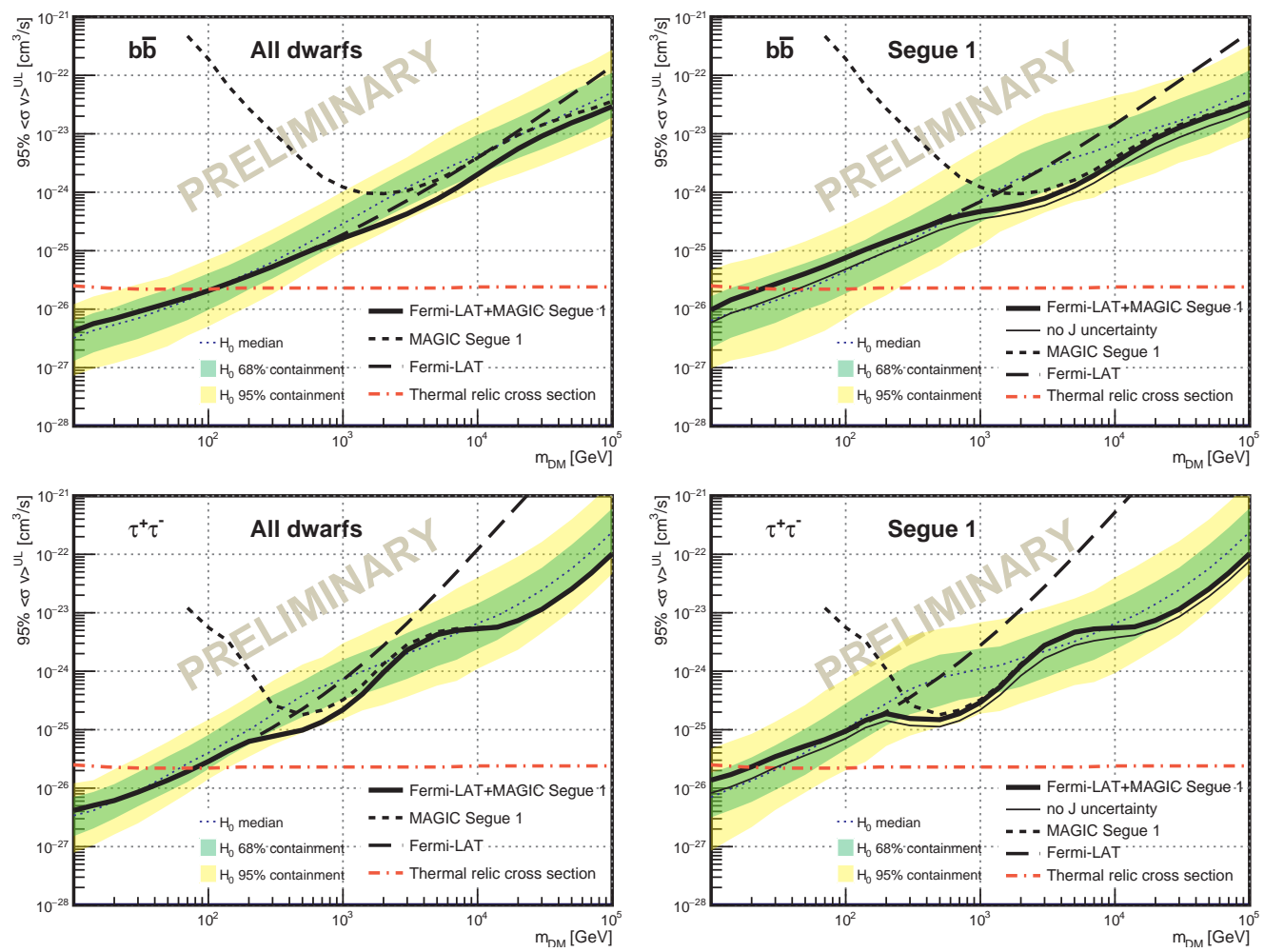

Figure 1: 95\% CL upper limits on the thermally-averaged cross section for DM particles annihilating into $b \bar{b}$ (upper plots) and $\tau^{+} \tau^{-}$(lower plots). Thick solid lines show the combined limits obtained by merging the Fermi-LAT observations of $15 \mathrm{dSphs}$ (left plots) or Segue 1 (right plots) with MAGIC observations of Segue 1. Dashed lines show the individual MAGIC (short dashes) and Fermi-LAT (long dashes) limits. J-factor statistical uncertainties are included. For the Segue 1 results, we also show (thin-solid line) the combined limits assuming a fixed J-factor (no statistical uncertainties). The thin-dotted line, green and yellow bands show, respectively, the median and the symmetrical, two-sided $68 \%$ and $95 \%$ containment bands for the distribution of limits under the null $\left(H_{0}:\langle\sigma v\rangle=0\right)$ hypothesis (see main text for more details). The red-dashed-dotted line shows the thermal relic cross section from [24].

\section{Discussion and Conclusions}

This work presents, for the first time, limits to the DM annihilation cross-section from a comprehensive analysis of gamma-ray data of energies between $500 \mathrm{MeV}$ and $10 \mathrm{TeV}$. Using a common, homogeneous analysis approach (both in the applied statistical methods and in the determination of the J-factors), we have combined the MAGIC observations of Segue 1 with Fermi-LAT observations of $15 \mathrm{dSphs}$. This allowed the computation of meaningful global DM limits, and the direct comparison of the individual results obtained with different instruments. Our results span the $\mathrm{DM}$ particle mass range from $10 \mathrm{GeV}$ to $100 \mathrm{TeV}$ - the widest range covered by a single analysis to date.

We have not observed any DM signal. Consequently, we set limits on the DM annihilation cross-section. Our results are the most constraining from observations of dSphs in the considered 
mass range. For the low-mass range, our results (fully dominated by Fermi-LAT data) are below the thermal relic cross-section $\langle\sigma v\rangle \simeq 3 \times 10^{-26} \mathrm{~cm}^{3} \mathrm{~s}^{-1}$. In the intermediate mass range (from few hundred $\mathrm{GeV}$ to few tens $\mathrm{TeV}$, depending on the considered annihilation channel), where FermiLAT and MAGIC achieve similar sensitivities, the improvement of the combined result with respect to the individual ones reaches a factor $\sim 2$. In addition, we present, for the first time, limits to high DM particle mass above $10 \mathrm{TeV}$ (fully dominated by MAGIC).

Our global analysis method is completely generic, and can be easily extended to include data from more targets, instruments and/or messenger particles provided they have similar sensitivity to the considered DM particle mass range. Of particular interest is the case of a global DM search from dSphs including data from all current gamma-ray (Fermi-LAT, MAGIC, VERITAS, H.E.S.S, HAWC) and neutrino (IceCube, Antares) instruments, and we hereby propose a coordinated effort toward that end. Including results obtained from other types of observational targets like the Galactic Center, galaxy clusters or others is formally also possible, but a common approach to the J-factor determination remains an open question. In the future, this analysis could include new instruments like CTA, Gamma-400 or Km3Net. Our global approach offers the best chances for indirect DM discovery, or for setting the most stringent limits attainable by these kinds of observations, therefore placing a new landmark in the field.

\section{Acknowledgments}

The MAGIC Collaboration thanks the Instituto de Astrofísica de Canarias for the excellent working conditions at the Observatorio del Roque de los Muchachos in La Palma. We also acknowledge the financial support of the ERDF under the Spanish MINECO (FPA2012-39502), of the CPAN CSD2007-00042 and MultiDark CSD2009-00064 projects of the Spanish ConsoliderIngenio 2010 program and of Centro de Excelencia Severo Ochoa SEV-2012-0234.

The Fermi-LAT Collaboration acknowledges support for LAT development, operation and data analysis from NASA and DOE (United States), CEA/Irfu and IN2P3/CNRS (France), ASI and INFN (Italy), MEXT, KEK, and JAXA (Japan), and the K.A. Wallenberg Foundation, the Swedish Research Council and the National Space Board (Sweden). Science analysis support in the operations phase from INAF (Italy) and CNES (France) is also gratefully acknowledged.

\section{References}

[1] A. Abramowski et al., Search for a Dark Matter annihilation signal from the Galactic Center halo with H.E.S.S. . Phys. Rev. Lett. 106 (2011) 161301. [arXiv: 1103 . 3266]

[2] J. Albert et al., Observation of Gamma Rays from the Galactic Center with the MAGIC telescope, Astrophys. J. 638 (2006) L101. [arXiv:astro-ph / 0512469$]$

[3] A. Archer et al., Very-High Energy Observations of the Galactic Center Region by VERITAS in 2010-2012, Astrophys. J. 790 (2014) 149. [arXiv: 1406 . 6383]

[4] M. Ackermann et al. Constraints on the Galactic Halo Dark Matter from Fermi-LAT Diffuse Measurements, Astrophys. J. 761 (2012) 91. [arXiv: 1205.6474]

[5] A. U. Abeysekara et al., The Sensitivity of HAWC to High-Mass Dark Matter Annihilations, Phys. Rev. D90 (2014) 122002. [arXiv: 1405 . 1730] 
[6] R. Abbasi et al. Search for Dark Matter from the Galactic Halo with the IceCube Neutrino Telescope, Phys. Rev. D84 (2011) 022004. [arXiv: 1101 .3349]

[7] S. Adrián-Martínez, Search of Dark Matter Annihilation in the Galactic Centre using the ANTARES Neutrino Telescope. [arXiv:1505.04866]

[8] J. Aleksić et al., MAGIC Gamma-Ray Observation of the Perseus Galaxy Cluster, Astrophys. J. 710 (2010) 634. [arXiv:0909.3267]

[9] A. Abramowski et al., Search for Dark Matter Annihilation Signals from the Fornax Galaxy Cluster with H.E.S.S , Astrophys. J. 750 (2012) 123. [arXiv: 1202.5494 ]

Erratum: ibid 783 (2014) 63

[10] T. Arlen et al., Constraints on Cosmic Rays, Magnetic Fields, and Dark Matter from Gamma-Ray Observations of the Coma Cluster of Galaxies with VERITAS and Fermi, Astrophys. J. 757 (2012) 123. [arXiv:1208.0676]

[11] M. Ackermann et al. Constraints on Dark Matter Annihilation in Clusters of Galaxies with the Fermi Large Area Telescope, JCAP 05 (2010) 025. [arXiv : 1002 . 2239]

[12] M. G. Aartsen et al. An IceCube Search for Dark Matter Annihilation in Nearby Galaxies and Galaxy Clusters, Phys. Rev. D88 (2013) 122001. [arXiv: 1307 . 3473]

[13] J. Aleksić et al., Optimized dark matter searches in deep observations of Segue 1 with MAGIC, JCAP 02 (2014) 008. [arXiv: 1312.1535]

[14] E. Aliu et al., Erratum: VERITAS deep observations of the dwarf spheroidal galaxy Segue 1 [Phys. Rev. D 85, 062001 (2012)]. Phys. Rev. D91 (2015) 129903(E)

[15] A. Abramowski et al., Search for dark matter annihilation signatures in H.E.S.S. observations of Dwarf Spheroidal Galaxies, Phys. Rev. D in press (2015). [arXiv: 1410 .2589]

[16] M. Ackermann et al., Searching for Dark Matter Annihilation from Milky Way Dwarf Spheroidal Galaxies with Six Years of Fermi-LAT Data. [arXiv: 1503.02641]

[17] J. Aleksić, J. Rico and M. Martinez, Optimized analysis method for indirect dark matter searches with Imaging Air Cherenkov Telescopes, JCAP 10 (2012) 032. [arXiv: 1209.5589 ]

[18] L. E. Strigari, Galactic searches for dark matter, Phys. Rep. 531 (2013) 1. [arXiv:1211. 7090 ].

[19] G. D. Martinez, A Robust Determination of Milky Way Satellite Properties using Hierarchical Mass Modeling. [arXiv:1309.2641]

[20] J. Aleksić et al., The major upgrade of the MAGIC telescopes, Part II: The achieved physics performance using the Crab Nebula observations, Astropart. Phys. in press (2015).

[arXiv:1409.5594]

[21] W. B. Atwood et al., The Large Area Telescope on the Fermi Gamma-ray Space Telescope Mission, Astrophys. J. 697 (2009) 1071.. [arXiv: 0902 . 1089]

[22] T. Sjöstrand et al. An Introduction to PYTHIA 8.2, Comput. Phys. Commun. 191 (2015) 159. [arXiv:1410.3012]

[23] J. F. Navarro, C. S. Frenk, and S. D. White, A Universal Density Profile from Hierarchical Clustering, Astrophys. J. 490 (1997) 493. [arXiv: astro-ph/9611107]

[24] G. Steigman, B. Dasgupta and J. F. Beacom, Precise relic WIMP abundance and its impact on searches for dark matter annihilation Phys. Rev. D86 (2012) 023506 